\title{
Les lésions de l'urètre associées à une fracture des corps caverneux. A propos d'une série de 4 cas et revue de la littérature
}

\author{
Hafed KETATA, Abdelkader BOUHLEL, Hammadi FAKHFAKH, Ahmed SAHNOUN, Ali BAHLOUL, \\ Mohamed Nabil MHIRI
}

Service d'Urologie, Hôpital H. Bourguiba, Sfax, Tunisie

\section{RESUME}

Introduction : Les lésions urétrales associées aux fractures des corps caverneux sont rares. Elles doivent être systématiquement recherchées. Leur traitement doit être chirurgical et entrepris en urgence.

Patients et méthodes : Nous avons colligé rétrospectivement, entre Janvier 1990 et Décembre 2004, 4 cas de rupture de l'urètre pénien parmi 96 cas de fractures des corps caverneux post-traumatiques, soit un taux de $4 \%$.

Résultats : l'âge moyen était de 36,5 ans (27-45 ans). Une urétrorragie a été observée chez tous les patients. Le traitement chirurgical en urgence a été réalisé dans tous les cas. La voie d'abord était une incision dans le sillon balano-préputial. Dans 2 cas, la rupture urétrale était partielle et associée à une fracture d'un seul corps caverneux.

Dans les 2 autres cas, il s'agissait d'une rupture urétrale totale associée à une fracture caverneuse bilatérale. L'intervention a consisté en une suture des corps caverneux et une réparation de l'urètre sur sonde tutrice. Après un recul moyen de 2,5 ans, deux cas de sténose courte de l'urètre, ne nécessitant pas de traitement chirurgical, ont été observées.

Conclusion : Le diagnostic de lésions de l'urètre associées à une fracture des corps caverneux est le plus souvent clinique. Les circonstances de l'accident sont très évocatrices. Un traitement chirurgical immédiat est le seul garant d'un résultat fonctionnel optimal.

Mots clés : corps caverneux, urètre, traumatisme, traitement chirurgical

\section{INTRODUCTION}

La fracture de la verge ou " faux pas du coït " est une pathologie relativement fréquente dans les pays du Maghreb. Son association à une rupture urétrale est rare. La lésion urétrale constitue un facteur pronostic aggravant. Les atteintes caverneuses et urétrales associées justifient une prise en charge chirurgicale urgente. La précocité du traitement détermine le devenir des fonctions mictionnelles et sexuelles des patients.

Entre janvier 1990 et décembre 2004, 96 patients présentant une fracture des corps caverneux ont été pris en charge. Parmi eux, 4 patients avaient une rupture urétrale associée. Dans la même période, 29 cas de rupture de l'urètre antérieure d'origine traumatique ont été observés. A travers quatre observations de rupture urétrale avec fracture des corps caverneux, nous étudions cette pathologie invalidante, cause possible de sténose urétrale et de troubles érectiles séquellaires.

\section{OBSERVATIONS ETUDIEES}

\section{Observation A}

Monsieur H.B., âgé de 45 ans, marié, ressent lors d'un faux pas du coït, une douleur vive de la verge avec un craquement et une détumescence immédiate suivis, quelque heure plus tard, d'une rétention aiguë d'urine.

Correspondance :

Dr Hafed KETATA - Service d'Urologie, Hôpital H. Bourguiba,

Avenue Magida Boulila 3029 Sfax. Tunisie -

Tel 0021698629129 - Fax 0021674241065 -

Email khafed@yahoo.fr 
A l'examen, il existe un hématome de la verge et du scrotum donnant une couleur violacée de la peau en regard, une incurvation gauche de la verge, une urétrorragie et un globe vésicale (Figure 1).

L'intervention chirurgicale est menée en urgence, après mise en place d'un cathéter sus-pubien (CSP), pour fracture de la verge avec rupture de l'urètre.

L'incision cutanée est circonférentielle, distale dans le sillon balano-préputial. Après retournement du fourreau pénien et évacuation de l'hématome, on découvre une déchirure de l'hémi-circonférence ventrale du corps caverneux droit, une petite fracture transversale du corps caverneux gauche et une rupture complète de l'urètre pénien, en regard de la lésion caverneuse (à 8 $\mathrm{cm}$ du méat urétral).

Les corps caverneux sont réparés par des points séparés (au fil fin $4 / 0$ à résorption lente). L'urètre est libéré sur $3 \mathrm{~cm}$ de part et d'autre de la rupture, cette libération est facilitée par la mise en place d'une sonde urétrale $\mathrm{CH}$ 18. On pratique une urétrorraphie termino-terminale par des points séparés (au fil fin $5 / 0$ à résorption lente). Le patient est mis sous antibiotiques et antiinflammatoires non stéroïdiens ainsi que du Diazépam pendant une semaine, pour éviter les érections involontaires.

Les suites opératoires sont simples : la sonde urétrale est enlevée au 7 ème jour, le drainage sus-pubien au 20ème jour post-opératoire. Le patient urine sans difficultés. L'urétrocystographie (UCR) de contrôle, au

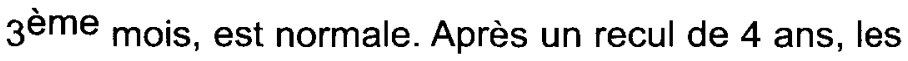
érections et la miction sont normales.

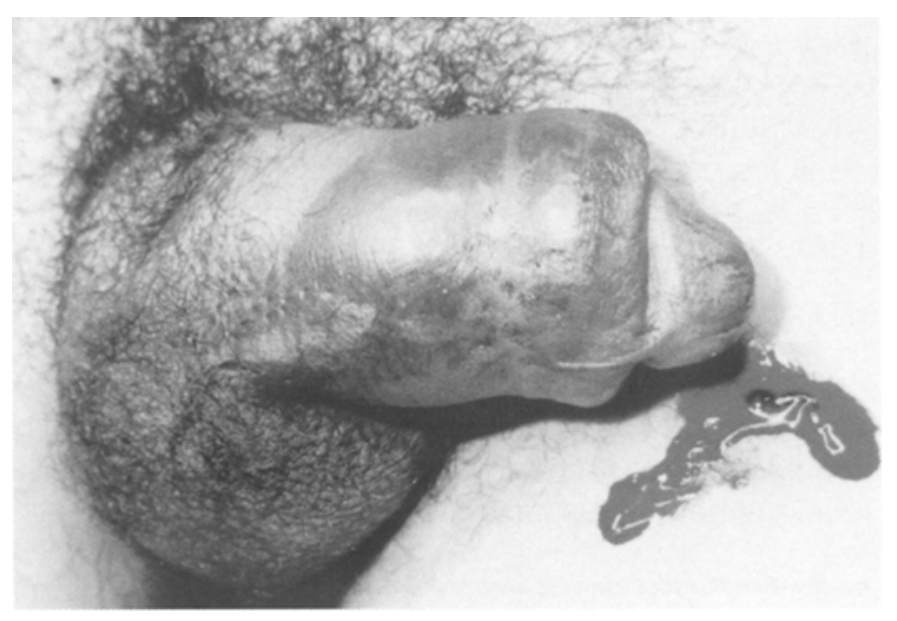

Figure 1: Hématome de la verge et du scrotum avec incurvation gauche de la verge avec urétrorragie.

\section{Observation B}

Monsieur M.S., âgé de 27 ans, célibataire, ressent au cours d'une manipulation forcée de la verge en érection, une violente douleur pénienne avec un craquement et une disparition instantanée de l'érection.

Al'examen, il existe un volumineux hématome pénien, une urétrorragie et un globe vésical.

L'intervention chirurgicale est menée en urgence, après mise en place d'un CSP. L'incision cutanée est circonférentielle, distale dans le sillon balano-préputial. Après évacuation de l'hématome, l'exploration trouve une déchirure transversale des deux corps caverneux avec une rupture complète de l'urètre, en regard de la lésion caverneuse, à $5 \mathrm{~cm}$ du méat urétral.

On procède à une réparation des corps caverneux et à une urétrorraphie termino-terminale, sur sonde urétrale $\mathrm{CH}$ 18. Du Diazépam est prescrit en postopératoire pendant une semaine ainsi qu'un traitement antibiotique et anti-inflammatoire non stéroïdiens.

Les suites opératoires sont simples : la sonde urétrale est enlevée au gème jour, le C.S.P au 21 ème jour. Les mictions reprennent normalement. L'UCR de contrôle au 3 ème mois est normale. A 3 ans, les érections et la miction sont normales.

\section{Observation $\mathrm{C}$}

Monsieur B.A., âgé de 31 ans, marié, ressent au cours d'une manipulation forcée de la verge en érection, une violente douleur de la verge avec un craquement et une disparition immédiate de l'érection suivis d'une dysurie tenace. A l'examen, la verge est incurvée vers la droite, siége d'un hématome sous cutané. II existe une urétrorragie minime.

L'intervention chirurgicale est menée en urgence pour fracture du corps caverneux gauche avec rupture de l'urètre. La voie d'abord est similaire aux cas précédents. L'exploration trouve une rupture du corps caverneux gauche avec une rupture partielle de l'urèthre pénien, en regard de la lésion caverneuse.

On procède à une réparation de l'albuginée du corps caverneux gauche puis à une urétrorraphie sur sonde urétrale $\mathrm{CH} 18$, par des points séparés au fil fin à résorption lente. Le patient est mis sous Etiléfrine pendant 15 jours pour éviter les érections et un traitement antibiotique et anti-inflammatoire non stéroïdiens pendant 7 jours.

Après ablation de la sonde urétrale, au 21 ème jour post opératoire, le patient reprend une miction normale. L'UCR à 3 mois montre un rétrécissement centré, peu serré et court de l'urètre pénien avec une perméabilité satisfaisante et un résidu post mictionnel négligeable. 
En l'absence de dysurie, une abstention thérapeutique avec surveillance clinique et par des débimètries a été décidée. A 2 ans, le patient n'a ni déformation, ni troubles de l'érection, ni dysurie.

\section{Observation D}

Monsieur M.F, âgé de 43 ans, marié, ressent lors d'un faux pas du coït, une douleur vive de la verge avec un craquement et une disparition immédiate de l'érection suivis, quelque heure plus tard, d'une rétention aiguë d'urine.

L'examen clinique trouve un hématome de la verge, une incurvation gauche de celle-ci, une urétrorragie et un globe vésicale. Une urétrographie faite en préopératoire pour préciser le siège exact de la lésion met en évidence une extravasation d'urines au niveau de la partie moyenne de l'urètre pénien (Figure 2).

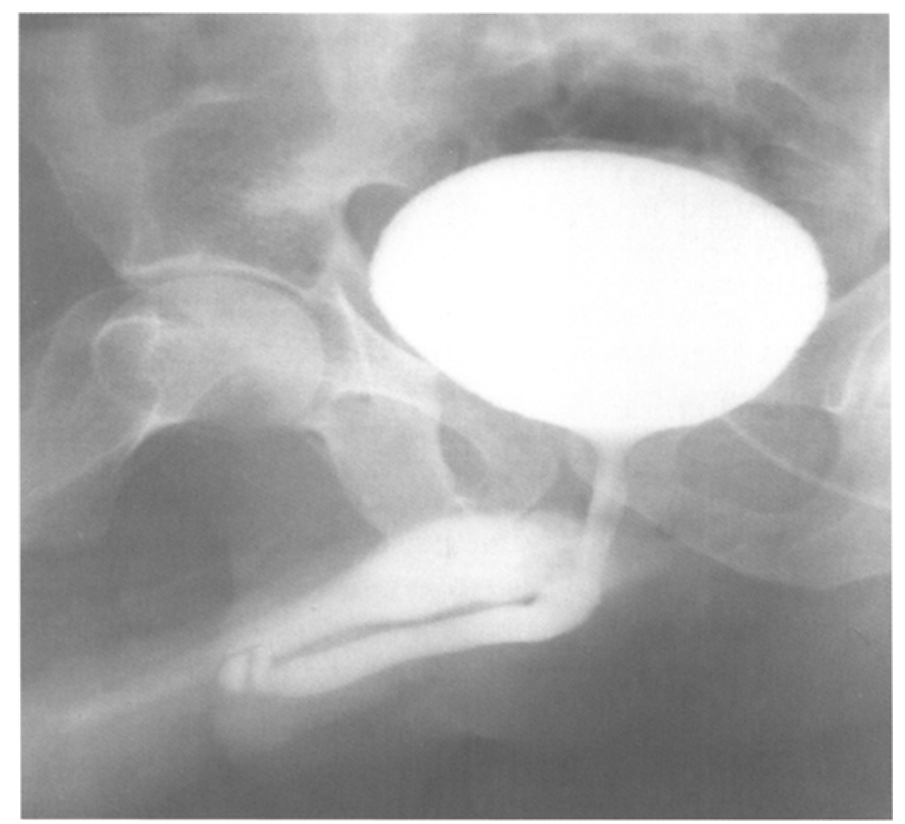

Figure 2 : Urétrocystographie antégrade et mictionnelle chez un patient admis pour fracture de la verge : lésion de la partie moyenne de l'urètre pénien.

L'intervention chirurgicale est menée en urgence, après mise en place d'un CSP. La voie d'abord est similaire aux cas précédents. II existe une déchirure de l'hémicirconférence ventrale du corps caverneux droit et une rupture partielle de l'urètre pénien, en regard de la lésion caverneuse. On procède à une réparation de l'albuginée du corps caverneux droit puis à une urétrorraphie, sur sonde urétrale $\mathrm{CH}$ 18. En post opératoire, un traitement par Etiléfrine pendant 2 semaines ainsi que des antibiotiques et des antiinflammatoires non stéroïdiens sont prescrits.
Les suites opératoires sont simples : la sonde urétrale est enlevée au $7^{\text {ème }}$ jour, le drainage sus-pubien au 21 ème jour. Les mictions reprennent sans difficultés. L'UCR à 3 mois, montre un rétrécissement centré, moyennement serré et court de l'urètre avec une perméabilité satisfaisante. On a opté pour des dilatations urétrales séquentielles rigides. A 1 an, le patient n'a ni déformation, ni troubles de l'érection, ni dysurie.

\section{DISCUSSION}

Les lésions urétrales secondaires à une fracture d'un ou des deux corps caverneux sont rares. Leur fréquence varie de 3 à $30 \%[4,12,20,25]$. Dans notre série, $4 \%$ des fractures de la verges étaient associées à une lésion urétrale. La fracture de la verge a été en cause dans $13 \%$ des cas de lésions de l'urètre antérieur nécessitant l'hospitalisation.

Cette affection touche le plus souvent l'adulte jeune entre 20 ans et 40 ans [11, 19], en pleine activité sexuelle, généralement célibataire $[4,5,9,10,11]$. La cause la plus fréquente, dans les pays du Maghreb, est la manipulation forcée et brutale de la verge en érection, souvent pour camoufler cette érection à l'entourage [3, $5,8,11,14,26]$. La lésion urétrale est plus fréquente en cas de faux pas du coït où la violence du traumatisme est plus importante qu'en cas de manipulation forcée [2].

Les ruptures urétro-caverneuse seraient favorisées par les infections gonococciques. Celles-ci, entraîneraient une fibrose et une diminution de l'élasticité de l'albuginée [20]. Le risque de rupture urétrale est majeur quand la verge en érection rigide subit une flexion dorsale à hauteur de l'angle péno-scrotal. Ce risque diminue à mesure qu'on se rapproche de l'extrémité distale de la verge $[11,16]$; la rupture urétrale est plus fréquente sur les faces ventrales et dorsales de l'urètre que sur les faces latérales. Elle siége au niveau de l'angle pénoscrotal dans plus de $50 \%$ des cas $[7,12,16,27]$.

La rupture urétrale est le plus souvent partielle. En cas de rupture complète, elle s'associe fréquemment à une fracture caverneuse bilatérale. La lésion urétrale siége généralement au même niveau que la lésion caverneuse $[3,5,11,12,16,24]$. Dans notre série, les deux cas de rupture urétrale complète étaient associés à une fracture caverneuse bilatérale.

Le diagnostic de rupture urétro-caverneuse est clinique. Les circonstances de survenue et la symptomatologie sont le plus souvent typiques [4]. La symptomatologie associe un tableau de rupture des corps caverneux (craquement, douleur vive, détumescence immédiate de la verge) et des signes faisant suspecter une lésion 
urétrale : urétrorragie (30 à $100 \%$ des cas) [16, 20, 26], miction douloureuse, difficile, voire impossible.

A l'examen, il existe le plus souvent une déviation de la verge du côté opposé de la lésion caverneuse. L'hématome est habituellement limité à la verge, il peut s'étendre au scrotum et au périnée en cas d'atteinte de l'intégrité du fascia pénis (fascia de Buck) $[3,12,16,17]$.

L'urétrorragie, signe habituellement la rupture de l'urètre. Certains auteurs, ont rapporté des cas d'urétrorragie sans lésions urétrales patentes $[17,20]$. Les troubles mictionnels peuvent s'observer sans atteinte urétrale. Ils seraient dus à des hématomes compressifs [26].

Les examens complémentaires ne sont pas indispensables pour le diagnostic [13]. Certains auteurs pratiquent une urétrographie rétrograde pour préciser le siége de la lésion urétrale $[5,12,15,16]$. L'imagerie par résonance magnétique des corps caverneux permet une bonne évaluation des lésions, notamment le diagnostic d'une lésion de l'urètre $[6,10,26]$. Son coût et sa disponibilité ne permettent pas son utilisation en pratique courante.

Les lésions urétrales peuvent êtres cliniquement latentes. Elles doivent être recherchées systématiquement en cas de fracture de la verge, notamment en peropératoire.

En cas de rupture urétrale complète, le traitement consiste en une réparation chirurgicale urgente de l'urètre et des corps caverneux [26]. La voie d'abord préférentielle est la voie circonférentielle distale, dans le sillon balano-préputial, avec retournement de la peau pénienne jusqu'au siège de la fracture. C'est une voie esthétique $[4,12,16,21]$, qui permet une bonne exposition des deux corps caverneux et de l'urètre. Elle est indiquée quand la fracture des corps caverneux est distale, bilatérale ou associée à une atteinte urétrale. Pour les lésions qui siégent au niveau de l'angle pénoscrotal et du tiers proximal de la verge, une incision médiane ventrale à hauteur de la lésion, permet un bon abord de l'urètre et de la face ventrale des corps caverneux $[16,26]$. Après évacuation de l'hématome et toilette de la plaie urétro-caverneuse, l'urètre est libéré sur $4 \mathrm{~cm}$ en amont et $4 \mathrm{~cm}$ en aval de la rupture. Ceci permet la réparation de l'albuginée au fil 4/0 à résorption lente jusqu'à la ligne médiane et la suture urétrale sans tension $[7,12,16,24,26]$.

Le drainage de l'urètre est assuré par une sonde multiperforée placée au niveau de l'urètre antérieur et laissée pendant 5 à 10 jours. Les urines sont dérivées par un drainage sus pubien laissé en place pendant 15 à 21 jours $[16,26]$.
En cas de rupture urétrale incomplète, certains auteurs préconisaient la dérivation des urines par cystostomie seule lorsque un traitement conservateur était envisagé pour la fracture de la verge $[1,17,22,23]$. Actuellement, la plupart des auteurs optent pour la réparation chirurgicale en urgence ; une prise en charge chirurgicale précoce des lésions caverneuses et urétrales permet de réduire le risque d'extravasation d'urines, d'angulation persistante de la verge, avec une diminution de la durée d'hospitalisation et une meilleure récupération de la fonction sexuelle $[16,26]$.

Concernant nos patients, le taux de sténose après réparation chirurgicale est de $50 \%$. Elles ont été observées après urétrorraphie pour des lésions urétrales partielles.

Le traitement médical d'appoint repose sur les antibiotiques et les anti-inflammatoires non stéroïdiens. Des tranquillisants ou une oestrogénothérapie sont le plus souvent indiqués pour prévenir les érections [16, 26].

En plus du suivi clinique, le contrôle, au $3^{\text {ème }}$ mois, par une urétrocystographie rétrograde et mictionnelle parait suffisant. II permet de déceler les sténoses urétrales [16, 26].

Non traitée, la fracture des corps caverneux évolue vers la formation d'un cal fibreux, responsable d'une angulation irréductible de la verge, de troubles érectiles et de douleurs au cours des rapports sexuels. Ce pronostic est plus grave en cas de lésion urétrale associée. Il existe un risque immédiat de rétention aiguë d'urine et d'infection de l'urohématome pouvant évoluer vers la formation d'un phlegmon et l'abcédation. Secondairement, l'évolution peut se faire vers l'apparition de fistules urétro-cutanées et de sténoses de l'urètre [5, $7,11,13,16,18]$.

\section{CONCLUSION}

Le diagnostic d'une lésion urétrale associée à une rupture des corps caverneux est clinique. Les circonstances de survenue et la symptomatologie sont le plus souvent typiques. La lésion urétrale constitue un facteur mettant en jeu le pronostic vital par le risque infectieux immédiat, et le pronostic fonctionnel par le risque de sténose urétrale et de fistule secondaires.

L'exploration chirurgicale immédiate doit rechercher systématiquement une lésion urétrale, le traitement permet de diminuer le taux de complications urinaires précoces et une reprise rapide d'une activité sexuelle normale. 


\section{REFERENCES}

1. AL SALEH B.M., ANSARI E.R., AL ALI I.H., TELL J.Y., SAHEB A. : Fractures of the penis seen in Abu Dhabi. J. Urol., 1985, 137: 274-275

2. ASGARI M.A., HOSSEINI S.Y., SAFARINEJAD M.R., SAMADZADEH B., BARDIDEH A.R. : Penile fractures : evaluation, therapeutic approaches and long-term results. J. Urol., 1996, $155: 148-149$.

3. BENCHEKROUN A., ABAKKA T., LAKRISSA A. : Fracture des corps caverneux. A propos de 22 cas. J. Urol. (Paris), 1986, 92: 291-295

4. BENNANI S., DAKIR M., DEBBAGH A., HAFIANI M., EL MOUSSAOUI A., EL MRINI M. et al. : La rupture traumatique du corps caverneux. Prog. Urol., 1998, 8 : 548-552.

5. BENNANI S., EL MRINI M., MEZIANE F., BENJELLOUN S. : La rupture traumatique du corps caverneux, A propos de 25 cas et revue de la littérature. Ann. Urol., 1992, $26: 355-359$.

6. BERGNER D.M., WILCOX M.E., FRENTZ G.D. : Fracture of the penis. Urology, 1982, $20: 278-280$.

7. BITKER M.O., BELIN J., JARDIN A., CHATELAIN C. : Faux pas du coit with associated rupture of corpora cavenosa and urethra. Urology, 1988, 32 : 447-448.

8. BOUJNAH H., RAKS S. : La fracture des corps caverneux. A propos de 67 cas. Ann. Urol., 1990, 24 : 313-315.

9. ELABD S., ABU FARHA O., EL GHARBAWY M., EL SHARABY M., EL MAHROUKY A. : Fracture of the penis and the result of surgical management.Injury. Br. J. Accident Surg., 1988, 19 : 381-383.

10. FEDEL M., VENZ S., ANDERSSEN R., SUDHOFF F., LOENING S.A. : The value of magnetic resonance imaging in the diagnosis of suspected penile fracture with atypical clinical findings. J. Urol., 1996, 155 : 1924-1927.

11. FERRAND J.Y., PHELINE Y. : Chirurgie réparatrice post traumatique des corps caverneux. J. Urol. (Paris), 1990, 96 : 111-113.

12. HAFIANI M., BENNANI S., DEBBAGH A., EL MRINI M., BENJELLOUN S. : Fracture bilatérale du corps caverneux avec rupture complète de l'urètre. J. Urol. (Paris), 1995, 101 : 200-202.

13. HARGREAVES D.G., PLAIL R.O. : Fracture of the penis causing a corporo-urethral fistula. Br. J. Urol., 1994, $73: 97$.

14. KOWALCZYK J., ATHENS A., GRIMALDI A. Penile fracture : an unusual presentation with lacerations of bilateral corpora cavernosa and partial disruption of the urethra. Urology, 1994, 44 : 599-601.

15. MAHARAJ D., NARAYNSINGH V. : Fracture of the penis with urethral rupture. Injury, 1998, $29: 483$.

16. MANGIN P., PASCAL B., CUKIER J. : Rupture de l'urètre par faux pas du coït. J. Urol. (Paris), 1983, $89: 27-34$.

17. MCANINCH J.W., KAHN R.I., JEFFREY R.B. : Major traumatic and septic genital injury. J. Traumatol., 1984, $132: 291-298$.

18. MOTIWALA H.G. : Urethrocavernous fistula following sexual intercourse. J. Urol., 1993, $149: 371$.

19. NARAYSINGH V., RAJU G.C. : Fracture of the penis. Br. J. Surg., 1985, $72:$ 305-306.

20. NICOLAISEN G.S. : Rupture of the corpus cavernosum : surgical management. J. Urol., 1983, $130:$ 917-919.

21. NOURI M., KOUTANI A., TAZI K. et al. : Les fractures du pénis à propos de 56 cas. Prog. Urol., 1998, $8: 542-547$.

22. ORVIS B.R., McANINCH J.W. : Penile rupture. Urol. Clin. North.Am., 1989, $16: 369-375$.
23. OZEN H.A., ERKAN J., ALKIBAY T., KENDI S., REMZI D. : Fracture of the penis and long term results of surgical treatment. Br. J. Urol., 1986, $58: 551-552$.

24. PAVARD D., GRISE P., DADOUN D. : Rupture complète de l'urètre et fracture bilatérale des corps caverneux. Ann. Urol., 1988, $22: 426-430$.

25. SARKIS P., DELMAS V., KOBELINSKY M., BOCCON GIBOD L. : Traumatismes de l'urèthre antérieur. E.M.C (Paris). Rein, organes génito-urinaires, 1993, 18-330, A10.

26. TOUITI D., AMEUR A., BEDDOUCH A., OUKHEIRA H. : La rupture de l'urètre au cours des fractures de la verge. A propos de 2 observations. Prog. Urol., 2000, $10: 465-468$.

27. TRIFA M., NJEH M., BAHLOULA., JEMAL S., MHIRI M.N. : Les ruptures traumatiques de l'urètre antérieur. A propos de 18 cas. Ann. Urol., 1997, $31:$ 313-317.

Manuscrit reçu : mars 2006 ; accepté avril 2006.

\section{ABSTRACT}

Urethral injury associated with corpora cavernosa fracture. Report of 4 cases and review of the literature

\author{
Hafed KETATA, Abdelkader. BOUHLEL, Hammadi \\ FAKHFAKH, Ahmed SAHNOUN, Ali BAHLOUL, \\ Mohamed Nabil MHIRI
}

Introduction: Urethral injury associated with corpora cavernosa fracture is a relatively uncommon form of urological trauma. Early diagnosis and immediate surgical repair are essential.

Patients and methods: Between January 1990 and December 2004, a total of 4 male patients with urethral injury associated with fracture of the corpora cavernosa were retrospectively studied from a series of 96 cases of penile fracture.

Results: The mean age of the patients was 36.5 years (range: 27-45 years). Urethral bleeding was noted in all patients. Immediate surgical repair via a penile circular subcoronal incision was performed in all cases. Two partial urethral injuries associated with a unilateral corpus cavernosum lesion were identified. In the other cases, total urethral injury was associated with bilateral rupture of the corpora cavernosa. The tunica albuginea defect was closed and the urethral laceration repaired over a urethral catheter. With a mean follow-up of 2.5 years, 2 short urethral strictures were observed and were managed non-operatively with good outcome.

Comments: Secondary urethral lesions associated with corpora cavernosa fracture most frequently affect young adults. 
In North African countries, forced self-manipulation of an erect penis is the most frequent cause, but the associated urethral lesion is usually related to vaginal intercourse.

The diagnosis can generally be made on the basis of history and physical examination alone. Blood at the meatus or haematuria or voiding difficulties suggest associated trauma to the urethra [4]. Additional imaging examinations are rarely required.

The standard treatment is prompt surgical exploration, evacuation of haematoma, and primary repair of the urethral and corpora cavernosa defects.

Late complications after surgical repair are penile curvature and pain during coitus. They are usually attributable to a residual fibrotic area.

Conclusion: Urethral injury associated with corpora cavernosa fracture usually has typical signs. The mechanism of trauma is very suggestive. Early surgical treatment is the only guarantee of a satisfactory functional result.

Key Words: corpora cavernosa, urethra, trauma, surgical treatment 